Methods Data pertaining to demographic characteristics, body parts discomforts and other occupational health related issues was collected from the users $(n=30$, localised in and around Guwahati city, India) of hand-held stone-polishing tool after administering the standardised questionnaire. Working postures was evaluated using REBA (rapid entire body assessment) technique. During use of polishing tool, quantification of the exposure to vibration was performed for 15 workers in three different working conditions (polishing on floor, stair and vertical wall). Following brain storming, CAD model and thereafter mock-up and prototype were developed from the final concept. Field trial of the redesigned polishing machine was carried out involving real users.

Results Following analysis and interpretation of the responses against the questionnaire, it was found that workers had been exposed to a high level of hand-arm vibration, adopting awkward postures, performing strenuous repetitive activities for prolong duration which ultimately imposed adverse impact on occupational health. Measurement of vibration transmission (in terms of intensity) to the wrist of the user before and after design intervention showed significant reduction.

Discussion The stone polishing machine was redesigned considering anthropometric and biomechanical compatibility to reduce biomechanical stress associated with hand and arm of the operators. A supportive mechanism was provided to avoid sustained load holding during polishing to reduce hand-arm fatigue. Handle grip was coated with rubber as vibration dampening material to reduce the vibration transmission to arm. The modified design with better usability and aesthetic look was liked by the users. It was evident from their higher comparative ratings against the existing design.

\section{GOOD WORK ABILITY OF THE UNEMPLOYED: ASSESSMENT BASED ON LENGTH OF UNEMPLOYMENT}

${ }^{1}$ Marja Hult* ${ }^{*}{ }^{1,2}$ Anna-Maija Pietilä, 'Terhi Saaranen. 'University of Eastern Finland, Kuopio, Finland; ${ }^{2}$ Kuopio Social and Healthcare Services, Kuopio, Finland

\subsection{6/oemed-2018-ICOHabstracts.1470}

Introduction Work ability of the unemployed is lower compared to their working counterparts and it significantly drops after two years of unemployment. This study aims to examine factors that are associated with good work ability based on length of unemployment.

Methods This study uses the data from 1981 unemployed or laid-off persons who responded to Finnish nationwide Regional Health and Well-being Study in 2014-2015. Logistic regression, adjusted for age and gender, was applied to analyse the association of several health and wellbeing factors, health behaviours, and work-related factors with good work ability, which was measured with Work Ability Score.

Result Short-term unemployed had significantly better work ability than long-term unemployed. For both groups, good self-rated health (short-term unemployed: OR 9.81, 95\% CI: 4.34 to 22.17; long-term unemployed: OR 9.69, 95\% CI: 3.93 to 23.40), high intense physical exercise (short-term: OR 4.27, 95\% CI: 1.64 to 11.16; long-term: OR 2.89, 95\% CI: 1.04 to 8.05 ), and belief in continuing to work until the retirement age (short-term: OR 11.75, 95\% CI: 5.10 to 27.09; long-term: OR 8.47, 95\% CI: 2.87 to 25.01$)$ were associated with good work ability. For short-term unemployed, a former employment status as a wage or salary earner (OR
4.62, 95\% CI: 1.45 to 14.76$)$; and for long-term unemployed, good mental health (OR 7.36, 95\% CI: 2.20 to 24.61) and good quality of life (OR 4.81, 95\% CI: 1.75 to 13.20), were also associated with good work ability.

Discussion Despite the length of unemployment, good work ability seems to associate strongly with a belief that person could continue working until the retirement age. Health is a substantial resource of work ability for both groups, but for long-term unemployed in particular, work ability is strongly involved in overall wellbeing and quality of life. The importance of physical activity should be highlighted when designing interventions for promoting work ability of unemployed persons.

\section{GENDER BASED VIOLENCE (GBV) AND OCCUPATIONAL SAFETY AND HEALTH CULTURE}

1,2,3,4 Khayongo Barbra Clara. 'International Commission on Occupational Health (ICOH); ${ }^{2}$ Allied Health Workers' Association, Kampala, Uganda; '3 Uganda Community and Occupational Health Association; ${ }^{4}$ Ministry of Gender, Labour and Social Development; Kampala, Uganda

\subsection{6/oemed-2018-ICOHabstracts. 1471}

Introduction Global evidence increasingly acknowledges the costs to GBV survivors and employers alike and the need for strategies to improve employees' safety at work and to reduce economic losses associated with employee's GBV perpetration or victimisation (Heise, et al., 2008). With the increasing strides by developing countries such as Uganda to achieve its mid-income status, there is increased efforts to boost the infrastructure sector though less focus has been put on balancing social risks and safety and health culture.

Study design; This was an explorative study that used qualitative data collection and analysis methods.

Results Amidst the high poverty indices and limited job security, workers in infrastructure endure all forms of violence ranging from verbal abuses, long working hours, sexual violence, working in hazardous environment and poor welfare services among other. The lack of grievance mediation modalities have exaggerated these violence thus contributing to increased fatal or non-fatal injuries, lost productivity time and damage to corporate image.

Discussion Sadly many forms of GBV in the workplace are not reflected in official records of employers neither are they rendered due justice by the police or courts of law. This lack of reporting is due to the lack of trust to exercise impartiality or some female workers feeling constrained to remain silent about their victimisation because of fear of reprisals against them, including the possibility of losing their livelihood.

This presentation seeks to explore Gender Based Violence and safety and health culture in the workplace and community.

\section{JOB INSECURITY AND PERCEIVED EMPLOYABILITY: A COMPARISON OF PUBLIC AND PRIVATE SECTOR}

H Koren, K Dežmar Krainz, K Bradvica, M Bubaš. Croatian Institute for Health Protection and Safety at Work, Zagreb, Croatia

10.1136/oemed-2018-ICOHabstracts. 1472

Introduction Job insecurity is one of the most important work stressors related to poor health and wellbeing. On the contrary, perceived employability is considered to be a resource increasing workers' control over working life and buffering 
negative outcomes of job insecurity. The aim of this study was to investigate the relationship between job insecurity, perceived employability and mental health among workers in public and private sector.

Methods A cross-sectional survey was conducted among 1038 Croatian workers from 6 different organisations. Healthcare and public transport service workers from public sector and industrial workers from private sector were included in this research. Correlational and regression analyses have been performed in SPSS.

Results Workers in public sector experience lower level of job insecurity and higher level of perceived employability in comparison to private sector workers. Job insecurity is related to poor mental health in both groups, while positive correlation of perceived employability and mental health was found only in public sector. Moderating effect of perceived employability on the relationship between job insecurity and mental health was found among public sector workers.

Discussion The results have shown significant differences in experiences of job insecurity, perceived employability and their relation to workers' mental health between sectors. In public sector, perceived employability was recognised as a moderator that can buffer the negative effects of job insecurity on mental health. This finding can have practical implications. In private sector, significantly higher level of job insecurity has been found, while there was no relationship between perceived employability and mental health. Possible explanation is that industrial workers are one of the most vulnerable groups in the labour market. On average they are less educated, dependant on their job, with fewer resources to cope job insecurity. Further investigation of other occupational groups within sectors should be conducted.

\section{A CROSS-SECTIONAL STUDY OF JAPANESE NON- PERMANENT WORKERS' MENTAL HEALTH}

Yusuke Noguchi*, Yumi Wakida, Haruka Ido, Ayako Hino, Kosuke Mafune, Hisanori Hiro. University of Occupational and Environmental Health, Japan, Kitakyushu, Japan

\subsection{6/oemed-2018-ICOHabstracts. 1473}

Introduction The proportion of atypical employment has increased in Japan since the economic crisis of the 1990s. Previous studies have reported that non-permanent employees tend to be psychologically distressed. However, the relationship between occupational stress and non-permanent workers' mental health is unclear. We investigated their job stress and psychological distress.

Methods We surveyed 86 non-permanent employees at a municipal office using the Effort-Reward Imbalance Questionnaire, Kessler 6 (K6), and Job Content Questionnaire (JCQ). Employees were divided into two groups based on an effortreward ratio (ER ratio) cutoff of 1, median reward scale scores (financial, esteem-related, and organisational reward), and median JCQ subscale scores (job demand, job control, and social support). We employed multi-way analysis of variance. In the first analysis, the dependent variable was the K6 score, and the independent variables were ER ratio, JCQ score, age, and gender. In the second analysis, we added three reward scales to the independent variables of the first analysis. The statistical significance level was set at 5\%.

Result In the first analysis, the main effect of ER ratio was marginally significant $[\mathrm{F}(1,75)=3.08, \mathrm{p}=0.0832]$. The least square mean of $\mathrm{K} 6$ scores was 5.54 in the high-ER ratio group and 3.28 in the low-ER ratio group. In the second analysis, no main effect was observed.

Discussion We hypothesised that non-permanent employees with low ER ratios would have better mental health than those with higher ER ratios. The result of the first analysis did not support this hypothesis, although it suggests this tendency. The second analysis showed no relationship between external reward and psychological distress. Thus, avoiding ER imbalance can lead to good mental health. The study limitations include the cross-sectional design and the lack of information about marital status and education level. Therefore, further investigation should be conducted.

\section{SYNERGISTIC EFFECTS IN INDUCING DAMAGE TO THE AUDITORY FUNCTION - COMBINED EFFECT OF SMOKING AND OCCUPATIONAL NOISE EXPOSURE ON HEARING LOSS}

${ }^{1} \mathrm{H} \mathrm{Hu},{ }^{1,2} \mathrm{~K}$ Kuwahara, ${ }^{3} \mathrm{~T}$ Nakagawa, ${ }^{3} \mathrm{~T}$ Honda, ${ }^{3} \mathrm{~S}$ Yamamoto, ${ }^{3} \mathrm{~T}$ Hayashi, ${ }^{1} \mathrm{~T}$ Mizoue. ${ }^{1}$ Department of Epidemiology and Prevention, National Centre for Global Health and Medicine, Japan; ${ }^{2}$ Teikyo University Graduate School of Public Health, Japan; ${ }^{3}$ Hitachi Health Care Centre, Hitachi Ltd., Japan

\subsection{6/oemed-2018-ICOHabstracts. 1474}

Introduction We aimed to prospectively assess the combined effect of smoking and occupational noise exposure on hearing loss.

Methods A prospective study was conducted using health check-up data collected between 2008 and 2015 in the Japan Epidemiology Collaboration on Occupational Health Study. The participants were 31444 employees, who were aged 3059 years, free of hearing loss at baseline. Smoking (never, former, or current) and occupational noise exposure (yes or no) were identified by a self-administered questionnaire at baseline. Major outcomes were high (hearing thresholds at $4 \mathrm{kHz}$ $>40 \mathrm{~dB}$ in at least one ear) and low (hearing thresholds at $1 \mathrm{kHz}>30 \mathrm{~dB}$ in at least one ear) frequency hearing loss. Cox proportional hazards model was used to estimate the combined effect of smoking and occupational noise exposure on hearing loss, adjusting for covariates.

Results During follow-up (a median of 6 years), 2101 individuals developed high-frequency hearing loss, and 979 developed low-frequency hearing loss. Compared with never smokers who reported no exposure to occupational noise, the hazard ratio (95\% confidence interval) for high-frequency hearing loss was $1.17(1.01,1.35)$ for past smokers without occupational noise exposure, $1.61(1.43,1.82)$ for current smoker without occupational noise exposure, $1.58(1.32,1.89)$ for never smokers with occupational noise exposure, $1.71(1.39,2.11)$ for past smokers with occupational noise exposure, 2.04 $(1.75,2.38)$ for current smoker with occupational noise exposure. For low-frequency hearing loss, the corresponding hazard ratios were $0.85(0.68,1.05), 1.08(0.91,1.29), 1.17$ (0.92, 1.49), $1.06(0.75,1.48), 1.42(1.13,1.78)$. Dose response relationship between smoking intensity and hearing loss was observed in both people with and without noise exposure.

Conclusion Smoking is a risk factor for hearing loss, independent of occupational noise exposure, and its combined effect on hearing loss with occupational noise exposure is additive. 